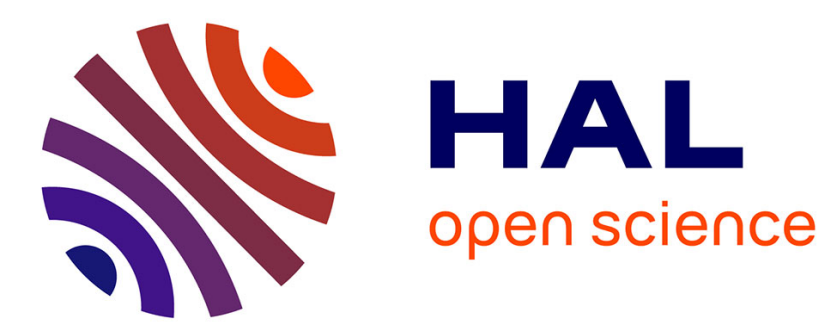

\title{
Le paradoxe Winnicott
}

\author{
Alain Vanier
}

\section{To cite this version:}

Alain Vanier. Le paradoxe Winnicott. Winnicott et la création humaine: Actes du colloque BB/ADOS, Oct 2010, Paris France. pp.39-42, 10.3917/lcp.151.0039 . hal-01522569

\section{HAL Id: hal-01522569 https://hal.science/hal-01522569}

Submitted on 7 Jun 2017

HAL is a multi-disciplinary open access archive for the deposit and dissemination of scientific research documents, whether they are published or not. The documents may come from teaching and research institutions in France or abroad, or from public or private research centers.
L'archive ouverte pluridisciplinaire HAL, est destinée au dépôt et à la diffusion de documents scientifiques de niveau recherche, publiés ou non, émanant des établissements d'enseignement et de recherche français ou étrangers, des laboratoires publics ou privés. 


\section{Le paradoxe Winnicott ${ }^{1}$}

\section{Alain VANIER}

Ce que tu viens de dire ne me rend pas la tâche facile, car c'est, pour une part, indiscutable. Il y a une dimension de témoignage, émouvante et précieuse, qui ne se discute pas. Mais un témoignage convoque toujours un tiers, devant qui attester, et, en t'écoutant, nous sommes mis à cette place - à tous points de vue excessive - qui ouvre au débat qui est un terme que tu aimes bien, d'autant que tu témoignes de ton débat avec Winnicott, avec son œuvre. Mon intervention sera plutôt un écho à ce que tu as pu apporter, plutôt qu'une discussion de propositions théoriques. En t'écoutant, je me suis demandé où j'étais en 1971, année fatale pour Winnicott. Ce mot de «liberté » que tu as rappelé, qui qualifiait l'attitude de Winnicott pour Octave Mannoni, m'a conduit à cette question. En 1971, j'entrais à peine dans mon premier stage de licence de psychologie à l'école de Bonneuil, fondée par Maud Mannoni avec l'aide, entre autres, de Pierre Fedida, qui adressait les étudiants de Paris 7 pour des stages bénévoles. Mais ce qu'on sait moins dans cette affaire, c'est la place de Winnicott dans cette 
aventure. En effet, Lacan avait adressé à Winnicott certains de ses élèves, Ginette Raimbault et Maud Mannoni qui entreprirent une supervision avec lui. Au cours de ses visites à Londres, Winnicott les avait encouragées à visiter Kingsley Hall, où travaillait Ronald Laing, qui avait été un de ses élèves. C'est là qu'est né le projet de créer un lieu dit d'« antipsychiatrie », c'est-à-dire en retenant «l'attitude » de cette expérience anglaise sans souscrire aux théories qui sous-tendaient le projet. C'est donc une des origines de la Fondation de Bonneuil. Je rappelle d'ailleurs que lors du Congrès de 1967 sur les psychoses de l'enfant, où, invité, Winnicott finalement ne vint pas, il envoya Laing et Cooper, ainsi qu'un texte La schizophrénie infantile en termes d'échec d'adaptation, traduit et lu par Octave Mannoni ${ }^{2}$.

J'insisterai aussi sur ce que Daniel Widlöcher rappelait, qui est essentiel, quant au peu de place fait à la psychanalyse d'enfant et au travail avec les psychotiques dans la formation des psychanalystes. Il témoigne, dans son livre Comment on devient psychanalyste...et comment on le reste ${ }^{3}$, de ce qui s'est passé en Angleterre avec la difficulté d'introduire l'analyse d'enfant dans la formation du psychanalyste. Que dire alors de la situation en France ! Mais, pour ouvrir la discussion, je retiendrai deux points, en commençant par la notion de paradoxe. Le paradoxe pour Winnicott n'est pas simplement quelque chose qui peut se déduire de son mode de pensée - une pensée qui se présente constamment comme paradoxale - mais un concept. Ce n'est pas seulement une tournure d'esprit, il en fait un concept.

Il est vrai que la pensée de Winnicott s'énonce très souvent sous cette forme, il y a certainement quelque chose de l'esprit anglais. Juliet Mitchell, lorsque nous avons fait ce colloque à l'Institut Français de Londres, Winnicott avec Lacan, s'étonnait de ce que les analystes français de tous bords s'intéressent tant à Winnicott ${ }^{4}$ : «He's so british! » En écoutant l'une des tables rondes précédentes, je pensais à cette idée, généralement admise aujourd'hui, qui nous vient d'outre-Manche, que nous sommes tous créateurs : a-t-elle la même résonnance dans le monde français? Dans un monde qui n'a pas eu par exemple quelqu'un comme l'évêque Berkeley dans sa culture. Il faut quand même un certain agencement de pensées dans une culture pour qu'ensuite d'autres pensées soient possibles. Ainsi Winnicott se disait très méfiant à l'égard des théories, mais on voit à quel point sa théorie est conséquente et consistante. Il prétendait même n'avoir pas lu Freud, mais tout déduire de l'expérience. Pourtant à certains endroits, il propose des commentaires précis de passages de textes de Freud. Une autre affirmation paradoxale ? Ou une méfiance toute britannique à l'égard d'un certain mode de théorisation?

Le paradoxe au sens de Winnicott n'est pas simplement une formule qui va à l'encontre de l'opinion commune, mais, sur le plan logique, un énoncé auquel on peut attribuer une valeur de vérité sans être amené à constater une contradiction. D'ailleurs, la psychanalyse n'est-elle pas fondamentalement paradoxale, puisqu'elle consiste à se défaire pour chacun de l'opinion commune ? A tel point, que, lorsque nos théories diffusant dans la culture finissent par nourrir le discours courant, elles deviennent le ferment majeur de la résistance commune à la psychanalyse ellemême, et ne sont pas sans poser de problème à l'expérience.

Mais le paradoxe a pour Winnicott une valeur de plus. Il donne au paradoxe une valeur fondamentale, conceptuelle, puisqu'il en distingue deux principalement qui renvoient à deux textes que Daniel Widlöcher a cités. Tout d'abord le « paradoxe essentiel », essential paradox ; et puis le second paradoxe, second paradox. Le paradoxe essentiel est celui de l'objet transitionnel. L'objet transitionnel est un objet que la mère propose à son enfant, s'attendant - dans la traduction française - à ce que l'enfant « s'y attache avec passion ». En anglais, la mère attend que l'enfant devienne addicted à cette chose ${ }^{5}$. Le lien est d'une nature très particulière, tout comme la position de l'autre dans l'investissement possible de l'objet, ce qui le situe dans un autre registre. Le paradoxe est que cet objet est à la fois à la mère et à l'enfant, et qu'en même temps, il n'est ni à l'un ni à l'autre. Ce n'est pas tant qu'il soit à l'un et à l'autre; c'est le fait qu'à la fois, il appartient aux deux et n'appartient pas à l'un ou à l'autre. A cet égard il joue le rôle d'intermédiaire, mais sa fonction propre échappe à l'observation. La séparation observable de deux individus ne correspond pas à ce qu'un analyste peut investir puisque, évidemment, il y a un intermédiaire, qui n'est pas qu'un objet médiateur, d'autant 
qu'il ne se situe pas au même niveau pour les deux. On ne saurait le réduire au tiers, puisqu'il ne se situe pas dans le même registre que les deux protagonistes, qui sont dans des positions fondamentalement dissymétriques.

Quant au "second paradoxe », il s'agit de l'expérience d'être seul en tant que nourrisson ou petit enfant en présence de la mère. Il y a d'autres paradoxes chez Winnicott, par exemple que l'objet est à la fois trouvé et créé - les résonances philosophiques, presque kantiennes, de la formule sont assez étonnantes. A ce niveau-là, Winnicott reste prudent. Dans ces cas, il ajoute « un philosophe ici pourrait dire quelque chose... », mais aussi « mais moi, je m'en distancie... » Il peut aussi décrire la déroute complète du philosophe, à qui il présenterait les idées qu'il est conduit à déduire de l'expérience. Il convient de remarquer que le premier paradoxe touche à l'objet, et le second paradoxe au sujet. Pour le second paradoxe, Winnicott déduit qu'il y a un passage de I am à I am alone, une évolution du « je suis » au « je suis seul ».

Le paradoxe, comme mode de conceptualisation privilégié de Winnicott, témoigne aussi d'apories qui sont celles que la psychanalyse introduit dans le langage quotidien, dans le langage courant, apories qu'il « convient d'accepter ». Ainsi l'objet transitionnel témoigne d'un espace, et ce qui est essentiel est sa dimension d'objet occulte, dimension qui n'est pas saisissable par l'observation. L'aire transitionnelle est fondamentale, et l'objet $\mathrm{y}$ fait écran, tout comme sa fonction dans le processus de séparation, car celle-ci, qui est son destin, a déjà eu lieu. Ces butées que le langage peine à saisir se manifestent aussi dans le saut que fait Winnicott quand il s'arrache à la stricte observation à cause de la psychanalyse. Il en parle dans un article de l'ouvrage dont Daniel Widlöcher a fait la critique lors de sa parution The Maturational Processes and the Facilitating Environment. Il y écrit que la psychanalyse procède en allant deeper and deeper, et l'observation earlier and earlier. Il insiste sur l'utilité de l'apport mutuel des deux démarches, l'observation de la psychologie du développement et la psychanalyse, à la condition de précautions épistémologiques qui ne soient pas oublieuses de la différence des registres temporels où elles se déploient.

Ma deuxième remarque, plutôt une question cette fois-ci, porte sur la copensée, notion qui t'est chère. Je ne suis pas compétent pour en parler, je ne suis même pas sûr d'être au clair avec sa définition, mais quand tu en as parlé, cela m'a évoqué le playing de Winnicott, ou quelque chose de cet ordre. A ce propos, il n'est pas inutile de mentionner la dimension maternelle du transfert chez Winnicott, sa position maternelle dans le traitement. Il le dit lui-même. Octave Mannoni pensait que c'était son point aveugle. C'est une question pour tout analyste et pour toute analyse, car c'est ce à quoi les analysants ont affaire surtout à la fin de la cure, au moment où l'analysant travaille les points aveugles de son analyste, là où celuici résiste fondamentalement. Si l'analyste veut bien entendre, en se dessaisissant de la place où le transfert l'assigne, il peut peut-être en apprendre quelque chose. D'où l'intérêt de poursuivre ce métier impossible.

Pour continuer surla copensée etle playing,je voudrais évoquer une hypothèse, construite à partir d'un travail ancien avec des bébés et leurs mères psychotiques, où Winnicott m'a servi de guide. Sur ce point, son article sur le cas Esther demeure l'article socle, le premier grand travail produit sur cette clinique. Mais, pour cela, il faut revenir à Melanie Klein, à ce qu'elle constate dans le cas Dick, quand elle dit qu'elle a été obligée de modifier sa technique en s'appuyant moins sur la production clinique que sur ses connaissances générales, entendons théoriques, pour fonder sa position d'analyste dans la cure et intervenir. Il me semble que, dans ces cas, l'analyste soutient une «supposition ». J'avais exposé les prémices de cette notion, «la supposition de sujet», dans un congrès de la WAIPAD il y a plus de vingt ans, à Lugano. La WAIPAD est l'ancêtre de la WAIMH, l'une des associations qui nous réunit aujourd'hui. Encore une réminiscence! C'est sans doute le style de l'exposé de Daniel Widlöcher qui les fait resurgir. Cette « supposition de sujet » n'est pas sans rapport avec la notion de holding chez Winnicott. Le holding de Winnicott n'est pas simplement un portage physique, et ne se réduit pas non plus au portage psychique au sens d'un contenant, où par exemple, la mère porterait psychiquement l'enfant. C'est plus que cela. Voilà un autre paradoxe que Winnicott propose : si le holding défaille, il ne dit pas que le nourrisson tombera par terre, il dit que le nourrisson tombera infiniment. On voit bien que l'on n'est plus du tout dans le champ de la réalité commune. Il ne cessera pas de tomber, mais en anglais c'est : il tombera infinitely. Quand 
on lit la transcription des enregistrements des proto-dialogues mère-bébé dans la période du turn talking on est frappé par la façon dont la mère interprète constamment les lallations du bébé. Elle est - pour reprendre le mot de Piera Aulagnier - son porteparole. Mais il me semble que, pour ce faire - et c'est une difficulté pour certaines mères il faut qu'elle suppose du sujet dans le bébé. Supposition, je ne l'entends pas comme certitude, mais plutôt croyance, une foi dans ce sujet comme continuité de ce support d'être. Et cette supposition est portée par la mère. Bien plus, il est créé par la mère. Le sujet est déjà là d'une certaine façon, mais il est dans la mère ; c'est elle qui le porte, elle est le premier lieu du sujet, cet Autre comme premier lieu du sujet. Ainsi l'agent de la création, ce n'est pas nous, pas le sujet, c'est l'Autre, déjà là, mais non encore constitué comme tel par le sujet. Celui-ci est dans la mère qui se représente le bébé comme séparé. C'est une modalité de l'anticipation. D'où la limite de l'observation qui voit deux individus, mais de quel point de vue ? Le sujet est dans la mère qui se le représente séparé et le fait ainsi exister, littéralement. D'où, pour certaines mères, ce que Winnicott remarquait pour Esther, une difficulté qu'elles rencontrent à pouvoir répondre à la demande quand elle émerge du côté de l'enfant d'une façon nouvelle. Quand l'enfant demande autre chose, ces mères sont en défaut et ne peuvent plus répondre. La demande suppose alors un élément détachable qui fait défaut, avec le corrélat du préalable de la reconnaissance d'un sujet que la mère peut figurer, avec qui elle n'a cessé d'ailleurs de parler avant même la naissance. Je pense à cette mère psychotique qui, quand on lui demandait pourquoi elle n'avait pas nourri depuis plusieurs heures son nourrisson d'une semaine ou de quinze jours qui pleurait, elle répondait : « Je n’ai pas faim. »

Je ne sais pas si cette hypothèse apporte quelque chose à la notion avancée par Daniel Widlöcher. C'est en tout cas un écho de ton intervention, et, peut-être, cet écho aura-t-il été l'occasion d'un exercice de l'ordre de la copensée ?

Alain Vanier Professeur de Psychopathologie à l'Université Paris Diderot, Psychanalyste Espace Analytique

\section{Références}

1- Transcription de la discussion de la conférence de Daniel Widlöcher au colloque BBados Winnicott et la création humaine, organisé par la WAIMHFrancophone (B. Golse), l'ISAPP-Francophone (A. Braconnier) et le Carnet Psy (M. Missonnnier), à Paris, Salle Gaveau, les 8 et 9 Octobre 2010.

2- Voir Enfance aliénée, Paris, Denoël, 1984.

3- D. Widlöcher, Comment on devient psychanalyste... et comment on le reste, Paris, Odile Jacob, 2010.

4- C. \&A. Vanier (dir.), Winnicott avec Lacan, Paris, Hermann, 2010.

5- « ...most mothers allow their infants some special object and expect them to become, as it were, addicted to such objects. » 